\title{
Two-stage acceleration of protons from relativistic laser-solid interaction
}

\author{
Jin-Lu Liu (刘晋陆), ${ }^{1}$ Z. M. Sheng (盛政明), ${ }^{1,2, *}$ J. Zheng (郑君), ${ }^{1}$ W. M. Wang (王伟民), ${ }^{2}$ M. Y. Yu (郁明阳), ${ }^{3,4}$ \\ C.S. Liu (刘全生), ${ }^{5}$ W. B. Mori, ${ }^{6}$ and J. Zhang (张杰) $)^{1,2}$ \\ ${ }^{1}$ Key Laboratory for Laser Plasmas (Ministry of Education) and Department of Physics, Shanghai Jiao Tong University, \\ Shanghai 200240, China \\ ${ }^{2}$ Beijing National Laboratory of Condensed Matter Physics, Institute of Physics, CAS, Beijing 100190, China \\ ${ }^{3}$ Institute for Fusion Theory and Simulation, Zhejiang University, Hangzhou 310027, China \\ ${ }^{4}$ Institute for Theoretical Physics I, Ruhr University, Bochum D-44780, Germany \\ ${ }^{5}$ East-West Space Science Center, University of Maryland, College Park, Maryland 20742, USA \\ ${ }^{6}$ Department of Physics and Astronomy and Department of Electrical Engineering, University of California,
} Los Angeles, California 90095-1547, USA

(Received 5 April 2012; published 24 October 2012)

\begin{abstract}
A two-stage proton acceleration scheme using present-day intense lasers and a unique target design is proposed. The target system consists of a hollow cylinder with conical inner wall, which is followed by the main target with a flat front and a dishlike flared rear surface. At the center of the latter is a tapered proton layer, which is surrounded by side proton layers at an angle to it. In the first acceleration stage, protons in both layers are accelerated by target normal sheath acceleration. The center-layer protons are accelerated forward along the axis while the side protons are accelerated and focused towards them. As a result, the side-layer protons radially compress as well as axially further accelerate the front part of the center-layer protons in the second stage. Two-dimensional (2D) particle-in-cell (PIC) simulations show that a quasimonoenergetic proton bunch with the maximum energy over $250 \mathrm{MeV}$ and energy spread $\sim 17 \%$ can be generated when such a target is irradiated with an 80 fs laser pulse with focused intensity $3.1 \times 10^{20} \mathrm{~W} / \mathrm{cm}^{2}$. Three-dimensional (3D) PIC simulation gives the reduced maximum energy $\sim 112 \mathrm{MeV}$ but even smaller energy spread $\sim 3 \%$ under the same laser conditions due to anisotropic electron acceleration with linearly polarized lasers.
\end{abstract}

DOI: 10.1103/PhysRevSTAB.15.101301

\section{INTRODUCTION}

Generation of high-energy well-collimated proton beams by relativistic intense lasers has attracted much interest in the past decade because its wide potential applications, such as proton oncology [1], medical isotope production [2], proton imaging [3], heavy-ion lithograph [4], as preaccelerated bunch for injection into conventional accelerators [5], fast-ion ignition for inertial confinement fusion [6], etc. For cancer therapy, one needs 200$250 \mathrm{MeV}$ proton beams with $\sim 1 \%$ energy spread and $\geq 10^{10} \mathrm{~s}^{-1}$ flux [7]. Although traditional accelerators can produce required proton beams, the high cost and large size makes it impractical for wide usage. With the rapid development of ultraintense short pulse laser technology [8], laser acceleration of charged particles has become an attractive alternative. Several schemes for generating energetic protons/ions from laser-solid interaction have been proposed, e.g., target normal sheath acceleration (TNSA)

\footnotetext{
*zmsheng@sjtu.edu.cn
}

Published by the American Physical Society under the terms of the Creative Commons Attribution 3.0 License. Further distribution of this work must maintain attribution to the author(s) and the published article's title, journal citation, and DOI.
PACS numbers: 52.38.Kd, 41.75.Jv, 52.50.Jm, 52.65.-y

[9-11], radiation pressure acceleration (RPA) [12], laser break-out afterburner (BOA) [13], Coulomb explosion acceleration [14], shock wave acceleration [15], hole boring acceleration [16], acceleration with mass-limited target [17], etc., as well as combinations of two or more of these schemes. So far, TNSA is the most experimentally investigated scheme [18]. In TNSA, protons at the rear surface of a solid target are accelerated by the sheath electric field of the laser-produced hot electrons there. Schemes for enhancing the energy and collimation of the proton beam have been proposed by using tailored targets that can focus the laser expelled electrons and thus enhance the sheath field and focus the accelerated protons [19]. However, the focused protons tend to diverge rapidly. Recent experimental and theoretical studies indicate that the proton energy can be enhanced by adding some kind of hollow cones at the front [20,21]. However, it is still rather difficult to produce $>100 \mathrm{MeV}$ proton beams using a planar target with TNSA. Ion acceleration with double layer targets by TNSA has been proposed [22] and further investigated theoretically and numerically in different parameter ranges [23]. Such targets provide the possibility to produce quasimonoenergetic proton beams, though the produced maximum proton energy does not change much from single layer targets. Theoretically, the RPA scheme can produce 
high-energy protons [12], however, extremely thin targets are required, which bring significant challenges for laser technologies, particularly on high contrast ratio at extremely high laser intensity. Taking into account these mentioned above, it is obvious that new practical and efficient schemes for proton acceleration under available experimental conditions are urgently demanded.

In this paper, we propose a scheme based upon a new target design, which can lead to two-stage acceleration of protons. Both two-dimensional (2D) and threedimensional (3D) particle-in-cell (PIC) simulations show that it can result in a well-collimated quasimonoenergetic proton beam with the peak energy over $100 \mathrm{MeV}$ by a readily available laser pulse at the focused intensity $\sim 3.1 \times 10^{20} \mathrm{~W} / \mathrm{cm}^{2}$.

\section{TARGET DESIGN}

The target is schematically shown in Fig. 1(a). The front part of the target is a metallic hollow cylinder with conical inner wall of cross section comparable to the laser spot. This front structure is followed by the main target slab, which has a flat front and dishlike rear back, and is a few micrometers thick at the center. The proton source on the rear surface of the slab consists of a tapered inner layer (the "I" protons) at the center, surrounded by specially shaped outer layers (the "O" proton) at an angle to the latter. When the target front is irradiated by an intense laser pulse, the conical inner wall at the front produces a large number of high-energy electron bunches (the " $C$ " electrons) [20,21,24], which are pushed forward along the cone wall by the laser ponderomotive force. These electron bunches propagate through the target and establish an intense electrostatic sheath field in the backside vacuum. The hollow cylinder outer wall also produce electron bunches (the " $\mathrm{H}$ " electrons) [25] propagating horizontally forward along the walls, which limit the transverse spread of the electrons from the conical inner wall and the main target slab. When the intense sheath field is established in the rear vacuum, protons from both source layers at the back of the target are accelerated by TNSA, this is the first acceleration stage.

The second acceleration stage is illustrated in Fig. 1(b). At initial time $t=t_{1}$, the I-protons (labeled " 1 ") from the inner or the center source layer are accelerated along the laser axis. The O-protons (labeled " 2 ") from the tilted outer source layers are accelerated towards the axis and eventually retarded by the fields of the propagating I-protons there. As a result, the I-proton bunch, which is elongating because of self-Coulomb repulsion (the faster I-protons are further accelerated at the expense of the slower ones), is laterally compressed by the obliquely converging O-protons at latter time $t=t_{2}$. Thus, the I-proton bunch is transversely compressed locally, and the fast I-protons at the front are additionally accelerated forward at the expense of the O-protons and the trailing I-protons. Because of this secondary

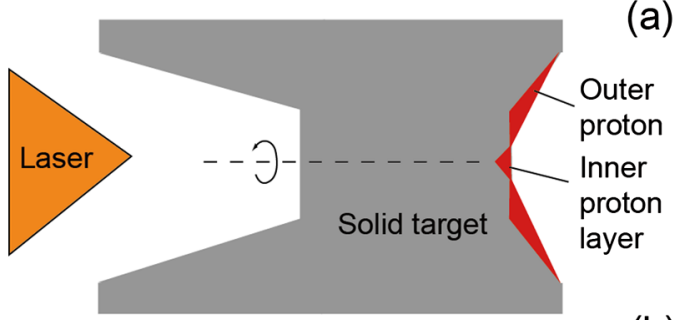

(a)
or
(b)

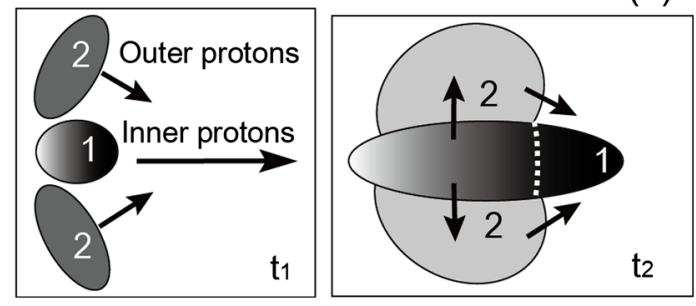

FIG. 1. (a) Sketch of the two-stage acceleration target, showing the target-front hollow cylinder with conical inner wall, the main target slab, which has a flat front and dishlike back side, where the tapered center and the two beveled outer proton layers are shown in red, and (b) schematic diagrams of the acceleration process.

acceleration, the fast I-protons in the front can gain much more energy, and eventually become detached and nearly monoenergetic. One can intuitively understand this process through a simple Coulomb interaction model. Assume that there are two charged bodies $A$ and $B$ moving forward at different velocities of $v_{A}$ and $v_{B}\left(<v_{A}\right)$ with an initial interval of $s$ between them and their mass and charge are given by $\left(m_{A}, q_{A}\right)$ and $\left(m_{B}, q_{B}\right)$, respectively. After an infinitesimal time $\Delta t$, the velocity increment for $A$ and the velocity reduction for $B$ is $\Delta v_{A}=k\left(\Delta t / m_{A} s^{2}\right)$ and $\Delta v_{B}=$ $\left(m_{A} \Delta v_{A} / m_{B}\right)$, respectively, where $k=q_{A} q_{B} / 4 \pi \varepsilon_{0}$. The corresponding kinetic energy changes for $A$ and $B$ are given by $\Delta \varepsilon_{A}=k\left(v_{A} \Delta t / s^{2}\right)$ and $\Delta \varepsilon_{B}=-k\left(v_{B} \Delta t / s^{2}\right)$, respectively. The kinetic energy increment of the two-body system is then $\Delta \varepsilon_{A}+\Delta \varepsilon_{B}=k\left[\left(v_{A}-v_{B}\right) \Delta t / s^{2}\right]=k \Delta s / s^{2}$, which exactly equals the reduction of the electric potential energy. Furthermore, the difference of the kinetic energies between $A$ and $B$ is $\Delta \varepsilon_{A}-\Delta \varepsilon_{B}=k\left[\left(v_{A}+v_{B}\right) \Delta t / s^{2}\right]$. This electrostatic repulsion mechanism happens between each O-proton and I-proton especially at their interface as shown in Fig. 1(b) (marked by white dotted curve). With the $\mathrm{O}$-protons and the trailing I-protons becoming integrated gradually, the distance between the head part I-protons and the main part O-protons becomes large, and this effect becomes weak ultimately. The above acceleration scenario is confirmed in our 2D and 3D PIC simulations.

\section{NUMERICAL SIMULATION RESULTS}

\section{A. 2D PIC simulation results}

We first present 2D PIC simulation results in detail. For the simulation we make use of the PIC code KLAP-2D with absorbing boundary condition along the longitudinal $(x)$ 

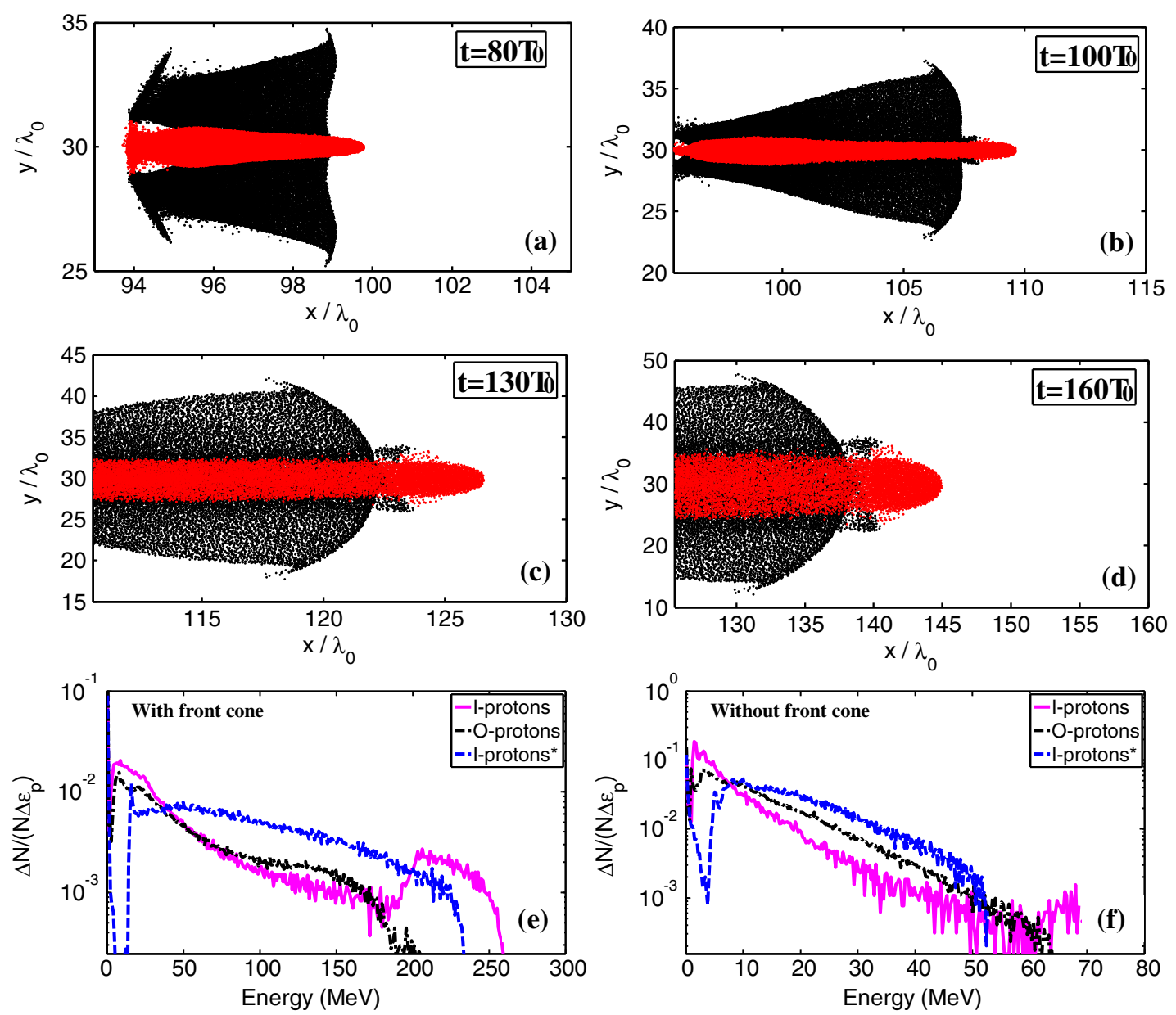

FIG. 2. 2D PIC simulation results of the spatial distribution of the I-protons (red dots) and O-protons (black dots) in the $x$ - $y$ plane at (a) $t=80 T_{0}$, (b) $t=100 T_{0}$, (c) $t=130 T_{0}$, and (d) $t=160 T_{0}$. The energy spectra for the I- and O-protons at $t=160 T_{0}$ are plotted in (e), where a spectrum marked as "I-protons*" is also plotted for the I-protons using the target without the O-proton layer initially for comparison and $N$ is the total number of I- or O-protons. The laser parameters are $a=12, \tau=30 T_{0}$, and $w_{0}=8 \lambda_{0}$. Plot (f) is obtained without the front cone.

direction and periodic boundary condition along the transverse $(y)$ direction for both fields and particles. The simulation box is $240 \lambda_{0} \times 60 \lambda_{0}$, where $\lambda_{0}=0.8 \mu \mathrm{m}$ is the laser wavelength, and it contains $14400 \times 2400$ cells. Each cell contains 100 numerical macroparticles in the plasma region. The simulation time step is $\Delta t=0.006 T_{0}$, where $T_{0}=2 \pi / \omega_{0}$ is the laser period. A $p$-polarized laser pulse of peak intensity of $I_{L} \sim 3.1 \times 10^{20} \mathrm{~W} / \mathrm{cm}^{2}$ [or $a=$ $e E_{L} /\left(m_{e} \omega_{0} c\right)=12$, where $E_{L}, e, m_{e}, \omega_{0}$, and $c$ are the peak laser electric field, the electron charge, the electron mass, the laser frequency, and the speed of light in vacuum, respectively] normally incident from the left side. The laser pulse has a normal Gaussian radial profile with the full width at half maximum (FWHM) $w_{0}=8 \lambda_{0}$ and a longitudinally Gaussian shape with the FWHM in $30 T_{0}$. The front of the laser pulse is initially at $x=40 \lambda_{0}$. The hollow cylinder and the main target slab consists of partially ionized plasma with effective charge-to-mass ratio $q / m_{i}=1 / 18360$. The front of the target is initially at $x=90 \lambda_{0}$, the ends of the $6^{\circ}$ open cone are $6.1 \lambda_{0}$ and $3 \lambda_{0}$ in diameter. The length and diameter of the hollow cylinder are $30 \lambda_{0}$ and $7.7 \lambda_{0}$, respectively. At the right end of the cylinder is the main target slab, as shown in Fig. 1(a). The thickness of the slab is $5 \lambda_{0}$. On its rear side, the thickness and transverse width of the tapered inner proton layer is $d=0.1 \lambda_{0}$ and $w=2 \lambda_{0}$, respectively. The maximum transverse and longitudinal thickness of the wedge-shaped outer proton layers are $0.3 \lambda_{0}$ and $0.9 \lambda_{0}$, respectively, and its angle relative to the axis is $70^{\circ}$. The charge-to-mass ratio of proton is $q / m_{p}=1 / 1836$. The electron density of the hollow cylinder, the cone, as well as the solid-target body is $n=100 n_{c}$, and that of both proton layers is $n_{p}=50 n_{c}$. Here $n_{c}$ is the critical density given by $\omega_{0}^{2}=4 \pi e^{2} n_{c} / m_{e}$. The initial temperature of heavy ions, protons, and electrons is assumed to be $1 \mathrm{keV}$ in all simulations.

Figure 2 shows snapshots of the spatial distributions of the I-protons (red dots) and the O-protons (black dots) in 
the $x-y$ plane at $t=80 T_{0}$ (a), $t=100 T_{0}(\mathrm{~b}), t=130 T_{0}$ (c), and $t=160 T_{0}$ (d). In Fig. 2(a) the I-protons are propagating along the axis, between the converging O-protons. As a result, the front part of the I-protons is further accelerated forward and the back part retarded, and the convergence of the O-protons slows down. Figure 2(b) shows that the upper and lower O-protons are closing up and merging with the trailing I-protons, whose front part is now further accelerated forward. The spatial distribution of the O-protons in fact has a bubblelike structure at the center because of the strong field of the I-protons inside the bubble. We see that the front of the O-protons is still concave near the center, that is, the O-protons are still converging towards the axis, squeezing and accelerating the front I-protons. Figure 2(c) shows that the front of the O-protons is flattened and the local pinching of the I-protons has resulted in a gap between the front running I-protons and the rest of the elongated bunch. Figure 2(d) shows that the gap broadens, indicating that the front running I-protons are detaching from the rest of the bunch. They form a $6 \mu \mathrm{m} \times 8 \mu \mathrm{m}$ nearly monoenergetic bunch. Figure 2(e) shows the energy spectra of the I-protons (red line) and the O-protons (black line) at $t=160 T_{0}$. One can see the quasimonoenergetic peak around $220 \mathrm{MeV}$ in the energy spectrum of the I-protons with the maximum energy $\sim 259 \mathrm{MeV}$ and energy spread $\sim 17 \%$, which is located just in front of the energy cutoff of the O-protons. We also plot the energy spectrum of the I-protons (blue line) under the condition initially without the O-proton layer for comparison. In this case, the I-protons have a broad energy spread with a lower maximum energy and there is no separated high-energy peak as found in the presence of the O-proton layer. This indicates obviously that it is the longitudinal pushing effect of the O-protons on the I-protons, which is responsible for the formation of the second stage acceleration and accordingly the quasimonoenergetic peak of the I-protons. The maximum proton energy in the case of having O-protons is enhanced by $11 \%$ compared with the case without O-protons in which the maximum proton energy is about $234 \mathrm{MeV}$.

To show the effect of the front hollow cone, we perform simulations with the same target design at the rear side but replacing the front cone with a normal planar surface. Figure 2(f) shows the produced energy spectra of the I-protons, the O-protons, and the case without O-protons. One notices that the maximum I-proton energy decreases significantly to less than $70 \mathrm{MeV}$. This can be attributed to the fact that the metallic hollow cone can provide a large number of $\mathrm{C}$-electrons and $\mathrm{H}$-electrons, which greatly enhances the electrostatic field at the target rear side as
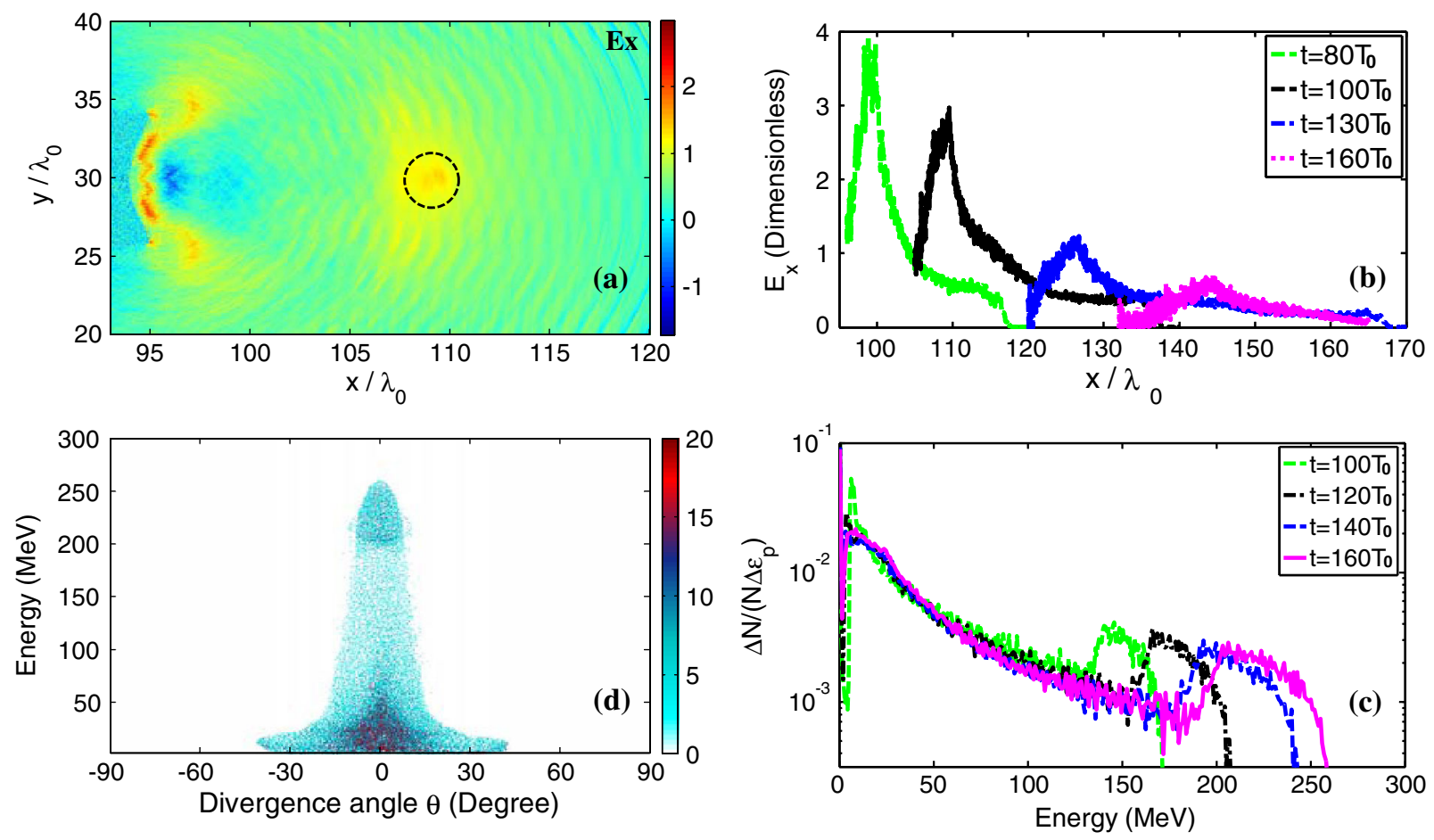

FIG. 3. 2D PIC simulation results of (a) spatial distribution of the axial electrostatic field $E_{x}$ (normalized by $m_{e} \omega_{0} c / e$ ) in the $x-y$ plane at $t=100 T_{0}$. An enhancement of the field at $x=108 \lambda_{0}$ can clearly be observed. (b) Evolution of the peak of $E_{x}$ on the axis near the interface between the front of the O-protons and the I-protons. Except for that for $t=80 T_{0}$, the $E_{x}$ values have been multiplied by a factor of 2 for clarity. (c) Evolution of the quasimonoenergetic I-proton peak. (d) Divergence angle and kinetic energy distribution of the I-protons at $t=160 T_{0}$; The laser parameters are the same as in Fig. 2. 
depicted above. One also notices that some high-energy I-proton presenting at the energy cutoff of the O-protons, which implies that the secondary acceleration also occurs in this case and it is mainly due to the initial structure of the proton layers at the rear target surface. Nevertheless, as shown in this figure, the target-front cone structure does help enhance the energy level, collimation, and acceleration distance of the resulting front I-proton bunch.

To further reveal the acceleration process, Fig. 3(a) shows the longitudinal electric field $E_{x}$ distribution in the $x-y$ plane at a relatively early time $t=100 T_{0}$. One can clearly see a strong positive electric field region (marked by the black dashed line in a circle) located around $x=$ $108 \lambda_{0}$, which is exactly at the interface between the I- and O-protons at that time, as shown in Fig. 2(b). The evolution of this space-charge electric field along the $x$ axis is shown in Fig. 3(b). The peak field moves forward with time, which enables the proton acceleration to occur in a long distance like a moving double layer in RPA [12], though its strength decreases with time rapidly. Consistent with this moving peak field, the quasimonoenergetic energy peak also extends to higher and higher energy. Figure 3(c) plots the energy spectra of the I-protons at $t=100 T_{0}, 120 T_{0}$, $140 T_{0}, 160 T_{0}$. The maximum proton energy at these times are $E_{\max }=171,207,243$, and $259 \mathrm{MeV}$, respectively. One notices that the energy width of the peak structure increases with the increasing of the maximum energy, while the distribution of the low energy part is almost unchanged. This explicitly indicates that the tail part of the I-protons has mixed with the O-protons, which pushes the fast I-protons together with the O-protons. Figure 3(d) shows that the fast I-protons have a small divergence angle located within the angle region $\theta_{\text {div }} \leq \pm 10^{\circ}$ and energy interval 200-260 MeV, where $\theta_{\text {div }}=\arctan \left(p_{y} / p_{x}\right)$ with $p_{x}$ and $p_{y}$ the longitudinal and transverse proton momentum, respectively. The average divergence angle, defined as $\theta_{\text {ave }}=\left(\sum\left(\theta_{\text {div }}\right)^{2} / N\right)^{1 / 2}$, is about $4.05^{\circ}$ for the energetic fast I-proton bunch. The number of the high-quality protons is about $8.7 \times 10^{11}$ (over $1 / 10$ of the total I-protons), which satisfies the requirement for many applications such as the cancer therapy.

In order to see how the laser parameters and the thickness of the inner proton layer affect the proton energy spectrum, we have carried out more simulations. Figure 4(a) shows the variation of the I-protons energy spectra obtained with the fixed laser energy of $9.6 \mathrm{~J}$ and $w_{0}=8 \lambda_{0}$ for $0.8 \mu \mathrm{m}$ laser, where the red curve is for $a=12$ and $\tau=30 T_{0}$ (case 1 , or $3.1 \times 10^{20} \mathrm{~W} / \mathrm{cm}^{2}$, $80 \mathrm{fs}$ ), the black curve is for $a=9$ and $\tau=53.3 T_{0}$ (case 2 , or $1.7 \times 10^{20} \mathrm{~W} / \mathrm{cm}^{2}, 140 \mathrm{fs}$ ), and the blue curve is for $a=15$ and $\tau=19.2 T_{0}$ (case 3, or $4.8 \times 10^{20} \mathrm{~W} / \mathrm{cm}^{2}, 50 \mathrm{fs}$ ). The maximum proton energy for case 1 is $259 \mathrm{MeV}$ as discussed before, while that for case 2 and case 3 are 174 and $322 \mathrm{MeV}$, respectively.
Therefore, the maximum proton energy scales with the peak laser amplitude like

$$
\mathcal{E}_{2 \mathrm{D}} \sim 2.1 a \sqrt{n / n_{c}}[\mathrm{MeV}],
$$

as depicted in Fig. 4(c), where $n$ is the electron density of the metallic target normalized by the critical density $n_{c}$. This is consistent with the fact that the high energy of hot electrons scales linearly with the laser amplitude $[16,24,25]$, no matter whether they are from the conical surface or the main target slab. Our simulation also indicates that the proton energy increases with the target density, which is typical with the TNSA mechanism [11]
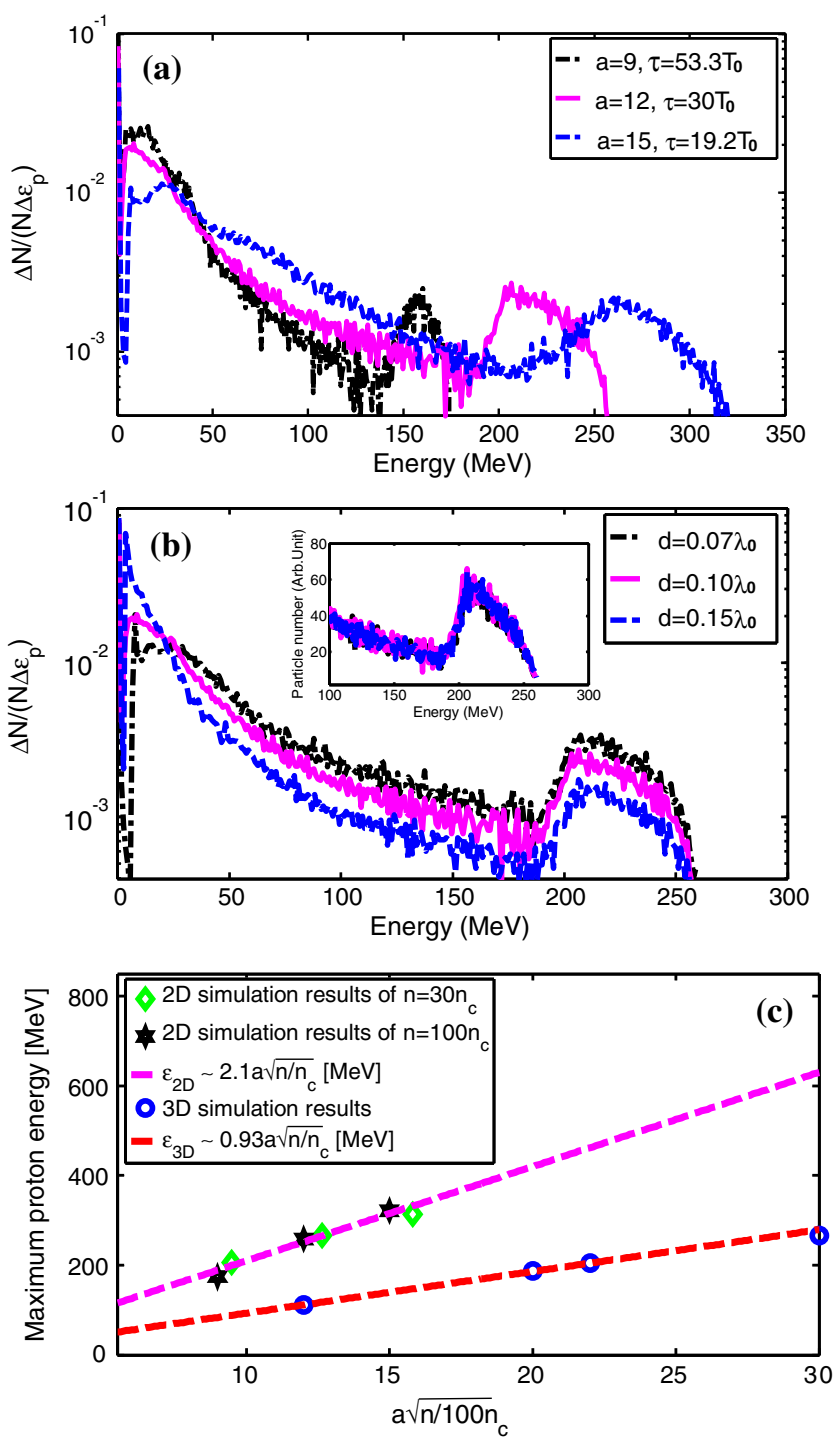

FIG. 4. 2D PIC simulation results of variation of the energy spectrum with respect to the laser amplitude $a$ and the laser pulse duration $\tau$ (a), and the thickness $d$ of the center proton layer (b). The laser energy is fixed to be $9.6 \mathrm{~J}$ and other laser parameters are the same as in Fig. 2. Plot (c) shows the maximum proton energy scaling as given by Eqs. (1) and (2) obtained in 2D and 3D PIC simulations. 
and can be attributed to the fact that fast electron number increases with the target density. The ratio of the proton numbers contained in the I-proton peak for three cases with peak laser amplitudes $a=9,12,15$ is about 0.41:1.0:2.73 and the ratio of the energy conversion efficiency is about $0.29: 1.0: 1.44$. We also performed 2D simulations of the same target design with the metallic target electron density as $n=30 n_{c}$ and the laser pulse with normalized electric field amplitudes $a=15,20$, and 25. The obtained maximum proton energy is 206,267 , and $313 \mathrm{MeV}$, respectively, which is also fairly well in line with the scaling of Eq. (1) as shown in Fig. 4(c).

Figure 4(b) shows the variation of the energy spectrum with respect to the thickness $d$ of the center proton layer. The red, black, and the blue curves are for $d=0.10 \lambda_{0}$, $d=0.07 \lambda_{0}$, and $d=0.15 \lambda_{0}$, respectively. Thus, the value of $d$ has a negligibly small effect on the maximum energy, the width of the energy spectrum, as well as the total number of protons in the high-energy peak. This is because the number of fast I-protons depends mainly on how and where the O-protons pinch the I-protons bunch and not on the total number of the I-protons. This novel character enables one to control the requested dose of the obtained proton bunch for applications.
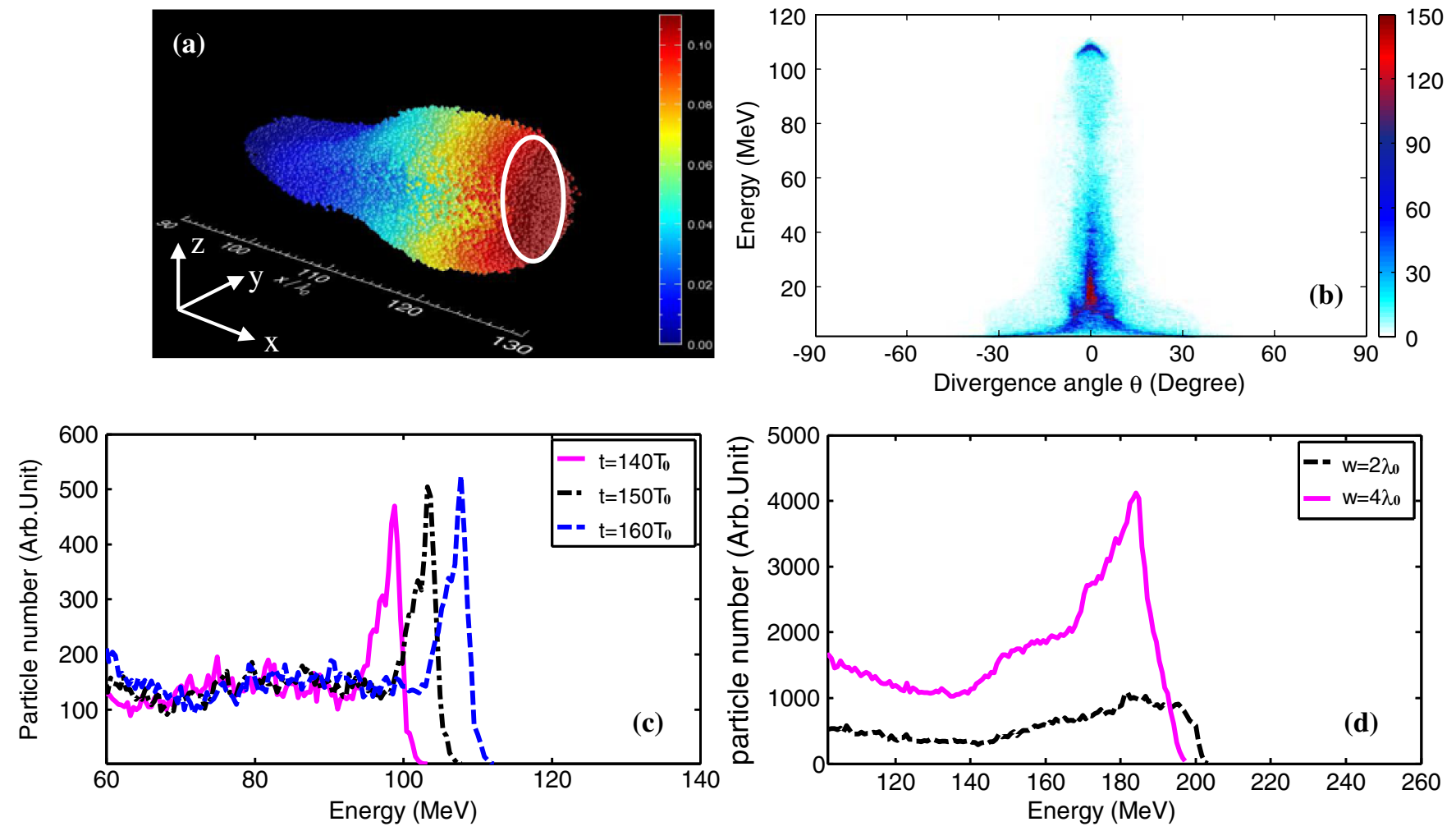

FIG. 5. 3D PIC simulation results. (a) Spatial and kinetic energy (normalized by $m_{p} c^{2}$ ) distribution of the I-protons at $t=160 T_{0}$. (b) Divergence angle and kinetic energy distribution of the I-protons at $t=160 T_{0}$. (c) Evolution of the energy spectra of the highenergy I-proton peak for $a=12$. (d) Comparison of the energy spectra of the high-energy part of the I-protons with its transverse size $w=2 \lambda_{0}$ (black dashed line) and $w=4 \lambda_{0}$ (pink solid line) obtained with the laser amplitude $a=22$ at $t=160 T_{0}$.
We also performed 3D PIC simulation with OSIRIS 2.0 [26] to check if this two-stage acceleration mechanism is effective in the real 3D geometry. The simulation box is $x \times y \times z=180 \lambda_{0} \times 60 \lambda_{0} \times 60 \lambda_{0}$ and it contains $14400 \times 480 \times 480$ cells. Each cell contains 64 numerical macroparticles in the plasma region. The simulation time step is $\Delta t=0.02 T_{0}$. The choice of the macroparticle number and the cell number in our simulation is limited by the available computer capability, however, it is accurate enough to make a judgement on the two-stage acceleration along the $x$ direction and is polarized in the $y$ direction. The target parameters are the same as in the 2D simulations if not specified.

Figure 5(a) plots the spatial distribution and kinetic energy (normalized by $m_{p} c^{2}$ ) of the I-protons at $t=$ $160 T_{0}$. It clearly shows that a condensed high-energy part is present in the central front of the I-protons (marked by the white line in a circle), where the color bar represents $\gamma-1$ with $\gamma$ is the Lorenz factor of the I-protons. Figure 5(b) shows the kinetic energy and divergence angle distribution of the I-protons at $t=160 T_{0}$, the color bar represents the relative proton number. One can see that effect with our target design. The incident laser propagates 
there exists a very narrow bright region at the top, which is the high-energy I-proton head as shown in Fig. 5(a). Figure 5(c) shows the evolution of the high-energy peak of the energetic I-protons. The maximum energy and peak energy slowly increase with time while the energy spread slightly decreases with time. One can see that a very narrow monoenergetic peak appears in the high-energy part of the I-protons, where the energy peak is $E_{\text {peak }}=$ $107.8 \mathrm{MeV}$, the maximum energy is $E_{\max }=112 \mathrm{MeV}$, the energy spread of this peak $\Delta E_{\mathrm{FWHM}} / E_{\text {peak }}$ is only about $3 \%$, and the proton number with kinetic energy larger than $100 \mathrm{MeV}$ is about $3.62 \times 10^{11}$. Comparing with the case without O-proton layers, in which the maximum energy of the I-protons is $76 \mathrm{MeV}$ and the energy spread is almost $100 \%$, the proton energy in the case with O-protons is enhanced by $47 \%$. This indicates that the pushing effect of the O-protons significantly improve the I-proton acceleration and lead to the formation of a quasimonoenergetic proton beam.

One notices that the high-energy proton beam obtained in the 3D case has two different points comparing with that in the 2D case: both the maximum energy and the energy spread in 3D case are considerably reduced as compared with those in the $2 \mathrm{D}$ case. The first is related to the inherent anisotropic electron acceleration with linearly polarized lasers in the 3D geometry. In this case, electrons from the front cone surface are mainly accelerated in the polarization plane (the $x-y$ plane) near the laser axis, i.e., the energetic electrons are elongated in the laser polarization (y) direction. Because of this anisotropic spatial distribution of hot electrons, they will diffuse in another transverse direction (the $z$ direction) later due to the space-charge field. As a result, the electrostatic fields at the rear surface are smaller than that in the $2 \mathrm{D}$ case. The anisotropic spatial distribution of hot electrons leads to anisotropic proton acceleration at the beginning, where the fast I-protons are also elongated along the $y$ direction. Such an asymmetry was also found recently in the 3D simulation of the BOA acceleration [27]. Later they will diffuse in the $z$ direction as shown in Fig. 5(a), which clearly shows that the spatial size along the $z$ direction is larger than that along $y$ direction. This leads to a thinner spatial distribution of the I-protons along the longitudinal $x$ direction and more uniform acceleration later in 3D case than those in $2 \mathrm{D}$ case. A supernarrow high-energy peak can thus be obtained.

To examine the scaling of proton energy with the laser intensity in the 3D geometry, we perform simulations using the laser amplitude $a=20,22$, and 30. The maximum proton energy of these obtained quasimonoenergetic proton bunches are $E_{\max }=187,204$, and $266 \mathrm{MeV}$, respectively. It turns out that the maximum proton energy scales with the peak laser amplitude like

$$
\mathcal{E}_{3 \mathrm{D}} \sim 0.93 a \sqrt{n / n_{c}}[\mathrm{MeV}],
$$

as also shown in Fig. 4(c). This scaling is similar to that obtained in 2D simulations except that the constant coefficient is smaller, which is caused by the three-dimensional effect as discussed above.

According to Eq. (2), higher energy protons can be produced with laser pulses at higher intensity. Let us take the case for the laser amplitude $a=22$, at which the maximum proton energy reaches about $204 \mathrm{MeV}$ at $t=$ $160 T_{0}$, as shown in Fig. 5(d). Even though this follows the scaling in Eq. (2), the resulting energy spectrum becomes very broad. Additional simulation indicates that one can reduce the energy spread simply by increasing the transverse size of the I-proton layer. When we take the transverse width of the I-protons $w=4 \lambda_{0}$, which is twice as large as used before, the energy spectrum is improved significantly as clearly revealed in Fig. 5(d). In this case, the maximum proton energy is comparable to the previous case and the proton number with kinetic energy larger than $170 \mathrm{MeV}$ is about $5.69 \times 10^{12}$. This can be understood as follows: a larger transverse width of the I-protons also means a larger inner diameter of the O-protons, which delays the merging time of the O-protons at the center. During this period, the high-energy part of the I-protons diffuses in the channel formed by the O-protons in a more uniform way transversely and gets more uniform acceleration inside the channel due to the longer O-protons merging time. This leads to a better monoenergetic I-proton peak. Also a longer O-proton merging time means a shorter pushing time in the second acceleration stage, which causes the smaller maximum proton energy in the case $w=4 \lambda_{0}$ as compared to the case $w=2 \lambda_{0}$, as shown in Fig. 5(d).

\section{SUMMARY}

In summary, we have proposed a two-stage acceleration target configuration for generating high-quality proton beams. With this target configuration, proton acceleration is accomplished by a two-stage process. In the first stage, TNSA produces two groups of protons with the first group moving forward from the center source layer and the second group moving toward the axis from the outer source layer. In the second stage, protons from the second group radially compress and axially push the front part of the first-group protons, realizing additional acceleration. 2D PIC simulations show that an 80 fs laser pulse with focused intensity $3.1 \times 10^{20} \mathrm{~W} / \mathrm{cm}^{2}$ can produce quasimonoenergetic proton bunch with maximum energy $\sim 259 \mathrm{MeV}$ and energy spread $\sim 17 \%$. 3D PIC simulations suggest that the proton energy scaling with the laser intensity is similar to that found in 2D simulation except for the difference in the constant coefficient. Even though the maximum energy found in $3 \mathrm{D}$ is reduced by half as compared to that in $2 \mathrm{D}$, the energy spread is also significantly reduced. With the same laser energy, higher laser intensity is beneficial for enhancing the maximum proton energy and the proton 
number in the quasimonoenergetic beam. With our scheme, the proton number in the quasimonoenergetic peak is over $10^{11}$ protons/shot. Moreover, the accelerated proton number is relatively stable even if one changes the rear proton layer thickness, laser intensity, and pulse duration. This also makes our scheme attractive for applications.

\section{ACKNOWLEDGMENTS}

Z.M.S. would like to acknowledge the OSIRIS Consortium, consisting of UCLA and IST (Lisbon, Portugal), for providing access to the OSIRIS 2.0 framework. This work is supported by the National Basic Research Program of China (Grant No. 2009GB105002) and the National Science Foundation of China (Grants No. 11121504, No. 10935002, and No. 11075105). C.S.L. is supported partially by the Overseas Master Program of the Ministry of Education of China. Numerical simulations have been performed partially on the supercomputer MAGIC CUBE at SSC Shanghai.

[1] A. R. Smith, Med. Phys. 36, 556 (2009).

[2] K. W. D. Ledingham et al., J. Phys. D 37, 2341 (2004).

[3] M. Borghesi, A. Mackinnon, D. Campbell, D. Hicks, S. Kar, P. Patel, D. Price, L. Romagnani, A. Schiavi, and O. Willi, Phys. Rev. Lett. 92, 055003 (2004); T. Sokollik, Springer Theses, 2011.

[4] B. Rout, A. D. Dymnikov, D. P. Zachry, E. V. Eschenazi, Y. Q. Wang, R.R. Greco, and Gary A. Glass, Nucl. Instrum. Methods Phys. Res., Sect. B 261, 731 (2007).

[5] K. Krushelnick et al., IEEE Trans. Plasma Sci. 28, 1184 (2000).

[6] M. Roth et al., Phys. Rev. Lett. 86, 436 (2001).

[7] S. S. Bulanov et al., Med. Phys. 35, 1770 (2008).

[8] G. A. Mourou, T. Tajima, and S. Bulanov, Rev. Mod. Phys. 78, 309 (2006).

[9] S. C. Wilks, A. B. Langdon, T.E. Cowan, M. Roth, M. Singh, S. Hatchett, M.H. Key, D. Pennington, A. MacKinnon, and R. A. Snavely, Phys. Plasmas 8, 542 (2001); A. Pukhov, Phys. Rev. Lett. 86, 3562 (2001).

[10] B. M. Hegelich, B. J. Albright, J. Cobble, K. Flippo, S. Letzring, M. Paffett, H. Ruhl, J. Schreiber, R. K. Schulze, and J. C. Fernández, Nature (London) 439, 441 (2006); H. Schwoerer, S. Pfotenhauer, O. Jäckel, K.-U. Amthor, B. Liesfeld, W. Ziegler, R. Sauerbrey, K. W. D. Ledingham, and T. Esirkepov, Nature (London) 439, 445 (2006).

[11] J. Fuchs et al., Nat. Phys. 2, 48 (2005); L. Robson et al., Nat. Phys. 3, 58 (2006); H. Daido, M. Nishiuchi, and A. S. Pirozhkov, Rep. Prog. Phys. 75, 056401 (2012).

[12] T. Esirkepov, M. Borghesi, S. V. Bulanov, G. Mourou, and T. Tajima, Phys. Rev. Lett. 92, 175003 (2004); X. Q. Yan, C. Lin, Z. Sheng, Z. Guo, B. Liu, Y. Lu, J. Fang, and J. Chen, Phys. Rev. Lett. 100, 135003 (2008); B. Qiao, M. Zepf, M. Borghesi, and M. Geissler, Phys. Rev. Lett. 102, 145002 (2009); M. Chen, A. Pukhov, T. P. Yu, and Z. M. Sheng, Phys. Rev. Lett. 103, 024801 (2009); A. P. L.
Robinson, M. Zepf, S. Kar, R. G. Evans, and C, Bellei, New J. Phys. 10, 013021 (2008); A. Macchi, S. Veghini, and F. Pegoraro, Phys. Rev. Lett. 103, 085003 (2009); V. K. Tripathi, C. S. Liu, X. Shao, B. Eliasson, and R.Z. Sagdeev, Plasma Phys. Controlled Fusion 51, 024014 (2009); H. B. Zhuo, Z. Chen, W. Yu, Z. Sheng, M. Yu, Z. Jin, and R. Kodama, Phys. Rev. Lett. 105, 065003 (2010).

[13] L. Yin, B. J. Albright, B. M. Hegelich, and J. C. Fernández, Laser Part. Beams 24, 291 (2006); L. Yin, B. J. Albright, B. M. Hegelich, K. J. Bowers, K. A. Flippo, T. J. T. Kwan, and J. C. Fernández, Phys. Plasmas 14, 056706 (2007);

[14] E. Fourkal, I. Velchev, and C. M. Ma, Phys. Rev. E 71, 036412 (2005).

[15] L. O. Silva, M. Marti, J. R. Davies, and R. A. Fonseca, Phys. Rev. Lett. 92, 015002 (2004); A. Macchi, F. Cattani, T. V. Liseykina, and F. Cornolti, Phys. Rev. Lett. 94, 165003 (2005); M. Chen, Z.-M. Sheng, Q.-L. Dong, M.-Q. He, Y.-T. Li, M. A. Bari, and J. Zhang, Phys. Plasmas 14, 053102 (2007); M. Q. He, Q.-L. Dong, Z.-M. Sheng, S.-M. Weng, M. Chen, H.-C. Wu, and J. Zhang, Phys. Rev. E 76, 035402(R) (2007); X. Zhang, B. Shen, X. Li, Z. Jin, F. Wang, and M. Wen, Phys. Plasmas 14, 123108 (2007); D. Haberberger, S. Tochitsky, F. Fiuza, C. Gong, R. A. Fonseca, L. O. Silva, W. B. Mori, and C. Joshi, Nat. Phys. 8, 95 (2011); C. A. J. Palmer et al., Phys. Rev. Lett. 106, 014801 (2011).

[16] S. C. Wilks, W. L. Kruer, M. Tabak, and A. B. Langdon, Phys. Rev. Lett. 69, 1383 (1992); H. Habara et al., Phys. Rev. E 70, 046414 (2004).

[17] T. Kluge, W. Enghardt, S. D. Kraft, U. Schramm, K. Zeil, T. E. Cowan, and M. Bussmann, Phys. Plasmas 17, 123103 (2010); T. Sokollik, M. Schnürer, S. Steinke, P. Nickles, W. Sandner, M. Amin, T. Toncian, O. Willi, and A. Andreev, Phys. Rev. Lett. 103, 135003 (2009); W. Yu, H. Xu, F. He, M. Yu, S. Ishiguro, J. Zhang, and A. Wong, Phys. Rev. E 72, 046401 (2005).

[18] K. Zeil, S. D. Kraft, S. Bock, M. Bussmann, T. E. Cowan, T. Kluge, J. Metzkes, T. Richter, R. Sauerbrey, and U. Schramm, New J. Phys. 12045015 (2010); J. Fuchs, P. Audebert, M. Borghesi, H. Pépin, and O. Willi, C.R. Physique 10, 176 (2009).

[19] T. Okada, A. A. Andreev, Y. Mikado, and K. Okubo, Phys. Rev. E 74, 026401 (2006); M. P. Liu, H.-C. Wu, B.-S. Xie, J. Liu, H.-Y. Wang, and M.Y. Yu, Phys. Plasmas 15, 063104 (2008); Y. Y. Ma, Z. M. Sheng, Y. Q. Gu, M. Y. Yu, Y. Yin, F. Q. Shao, T. P. Yu, and W. W. Chang, Phys. Plasmas 16, 034502 (2009).

[20] S. A. Gaillard, T. Kluge, K. A. Flippo, M. Bussmann, B. Gall et al., Phys. Plasmas 18, 056710 (2011); T. Kluge, S. A. Gaillard, K. A. Flippo et al., New J. Phys. 14, 023038 (2012).

[21] Y. Nodera, S. Kawata, N. Onuma, J. Limpouch, O. Klimo, and T. Kikuchi, Phys. Rev. E 78, 046401 (2008); W. M. Zhou, Y. Gu, W. Hong, L. Cao, Z. Zhao, Y. Ding, B. Zhang, H. Cai, and K. Mima, Laser Part. Beams 28, 585 (2010); J. Zheng, Z.-M. Sheng, J.-L. Liu, W.-M. Zhou, H. Xu, and J. Zhang, Phys. Plasmas 18, 113103 (2011).

[22] T. Zh. Esirkepov et al., Phys. Rev. Lett. 89, 175003 (2002). 
[23] S. S. Bulanov et al., Phys. Rev. E 78, 026412 (2008); A. V. Brantov, V.T. Tikhonchuk, V. Yu. Bychenkov, and S.G. Bochkarev, Phys. Plasmas 16, 043107 (2009).

[24] Z. M. Sheng, Y. Sentoku, K. Mima, J. Zhang, W. Yu, and J. Meyer-ter-Vehn, Phys. Rev. Lett. 85, 5340 (2000); Y. T. Li et al., Phys. Rev. Lett. 96, 165003 (2006); M. Chen, Z.-M. Sheng, J. Zheng, Y.-Y. Ma, M. Bari, Y.-T. Li, and J. Zhang, Opt. Express 14, 3093 (2006).
[25] Y. Y. Ma, Z.-M. Sheng, Y.-T. Li, W.-W. Chang, X.-H. Yuan, M. Chen, H.-C. Wu, J. Zheng, and J. Zhang, Phys. Plasmas 13, 110702 (2006); F. C. Wang, B. Shen, X. Zhang, Z. Jin, M. Wen, L. Ji, W. Wang, J. Xu, M. Y. Yu, and J. Cary, Phys. Plasmas 16, 093112 (2009).

[26] R. A. Fonseca et al., Lect. Notes Comput. Sci. 2331342 (2002).

[27] L. Yin, B. Albright, K. Bowers, D. Jung, J. Fernández, and B. Hegelich, Phys. Rev. Lett. 107, 045003 (2011). 